\title{
Synthesis of Side-Chain-Free Hydrazone-Linked Covalent Organic Frameworks through Supercritical Carbon Dioxide Activation
}

\author{
Shashini D. Diwakara ${ }^{a}$ (D) \\ Gregory T. McCandless ${ }^{a}$ \\ Sampath B. Alahakoon ${ }^{b}$ (D) \\ Ronald A. Smaldone*a (iD) \\ a The Department of Chemistry and Biochemistry, The University of Texas at Dallas, \\ Richardson, Texas 75080, United States \\ ${ }^{b}$ Institute for Combinatorial Advanced Research \& Education (KDU-CARE), General Sir \\ John Kotelawala Defence University Kandawala Rd, Ratmalana, 10390, Sri Lanka \\ ronald.smaldone@utdallas.edu
}

Received: 16.02.2021

Accepted after revision: 15.03.2021

DOI: 10.1055/a-1477-5123; Art ID: om-21-0021oa

License terms: CCO

(c) 2021. The Author(s). This is an open access article published by Thieme under the terms of the Creative Commons Attribution-NonDerivative-NonCommercial License, permitting copying and reproduction so long as the original work is given appropriate credit. Contents may not be used for commercial purposes, or adapted, remixed, transformed or built upon. (https://creativecommons.org/licenses/by-nc-nd/4.0/)

Abstract Supercritical carbon dioxide $\left(\mathrm{scCO}_{2}\right)$ activation provides milder conditions to process covalent organic frameworks (COFs) without compromising their crystallinity and porosity. To this end, three hydrazone COFs (TFPB-DHz COF, TFPT-DHz COF, Py-DHz COF) were synthesized with a terephthaloyl dihydrazide linker $(\mathbf{D H z})$ which has no substituents. To date, the synthesis of hydrazone COFs without a narrow range of alkoxy linkers has not been possible. The $\mathrm{scCO}_{2}$-activated hydrazone-linked COFs in this study were crystalline and had high surface areas (surface areas of TFPB-DHz COF, TFPT-DHz COF, and PyDHz COF were 790, 1199, and $932 \mathrm{~m}^{2} / \mathrm{g}$, respectively). This study shows the significance of using milder activation methods for making hydrazone-linked COF structures that were previously inaccessible.

Key words covalent organic frameworks, hydrazones, supercritical carbon dioxide

\section{Introduction}

Covalent organic frameworks (COFs) are a class of crystalline, porous, and two-dimensional (2D) or threedimensional organic polymer materials. They are composed of lightweight elements $(\mathrm{C}, \mathrm{H}, \mathrm{O}, \mathrm{N}, \mathrm{B})$ and synthesized via dynamic covalent bond formation. ${ }^{1-3}$ Different types of linkages such as imine, ${ }^{4}$ hydrazone, ${ }^{5}$ azine, ${ }^{6}$ boronate ester, ${ }^{1}$ and triazines ${ }^{7}$ can be found in these materials. After seminal work by Yaghi and co-workers in $2005,{ }^{1}$ numerous COFs have been developed. These materials have been employed for various applications including catalysis, ${ }^{8}$ gas storage, ${ }^{9}$ gas separations, ${ }^{10}$ energy storage, ${ }^{11}$ and sensing, ${ }^{12}$ due to their promising properties such as well-defined structures, ${ }^{3,13}$ high surface area, ${ }^{9}$ tunable pore size, ${ }^{14}$ and low density. ${ }^{9}$

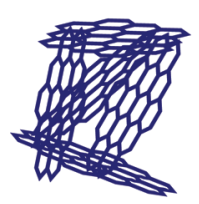

Amorphous XNon-Porous
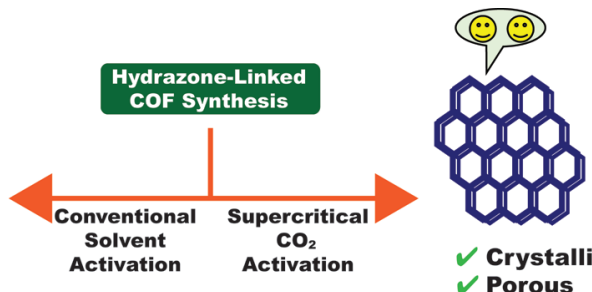

$\checkmark$ Crystalline

$\checkmark$ Porous
The general mechanisms of COF polymerization and assembly have received considerable attention over the past few years ${ }^{15,16}$ in order to help researchers understand why certain combinations of monomers form crystalline COFs over others. Hydrazone-based COFs have been studied extensively due to their physicochemical stability compared to imine, or boronate ester-linked COFs. ${ }^{5,17,18}$ 2,5-Diethoxyterephthalohydrazide was used as the hydrazide linker in the first report of hydrazone-linked COFs by Yaghi and coworkers. ${ }^{5}$ Since then many other hydrazone COFs have been reported using this strategy. ${ }^{17,19-21}$ In almost all these reports, hydrazone COFs were synthesized with monomers containing alkoxy (methoxy, ethoxy, propoxy) or allyloxy side chains. ${ }^{17,20-22}$ However, the role of these side chains in the initial polymerization of hydrazone COFs has not been fully elucidated. It is clear that these side chains play a more crucial role in the formation of crystalline materials than in COFs with other types of linkages such as imines, $\beta$-ketoenamines, or boronate esters. Attempts to synthesize hydrazone COFs from unsubstituted or alkyl chain substituted hydrazides have so far been unsuccessful and typically result in non-porous or amorphous polymers (or both). ${ }^{19,20,22}$ Previous studies have reported the importance of these side chains through mechanistic and spectroscopic studies and found that these side chains can facilitate the formation of intramolecular hydrogen bonding, which causes the restriction of intramolecular bond rotation. ${ }^{20,22,23}$

As shown in Figure 1a and b, the presence of alkoxy side chains enables improved intra- and interlayer hydrogen bonding. In contrast, monomers without any substituent can experience free intramolecular bond rotation resulting in more flexibility in the sheet structure that can potentially reduce crystallinity. If these side chains serve to rigidify the 2D layers or enhance the strength of the interactions between the sheets, then it is possible that the lack of these side chains could also reduce the stability of the COFs to activation. Indeed, previous studies have shown that the method of activation used on a COF plays a pivotal role in its bulk porosity and crystallinity. The most widely used 
A

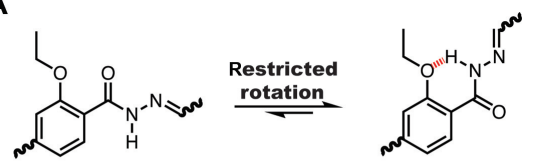

B

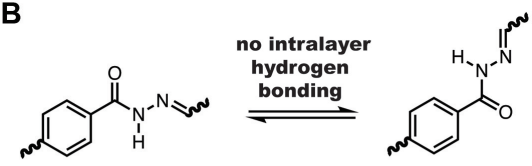

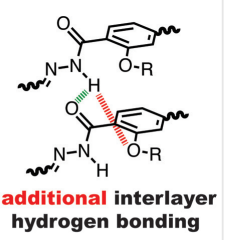

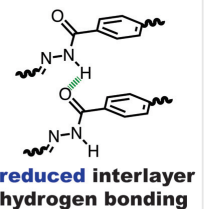

C

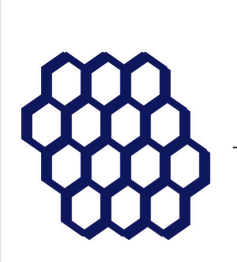

Crystalline COF

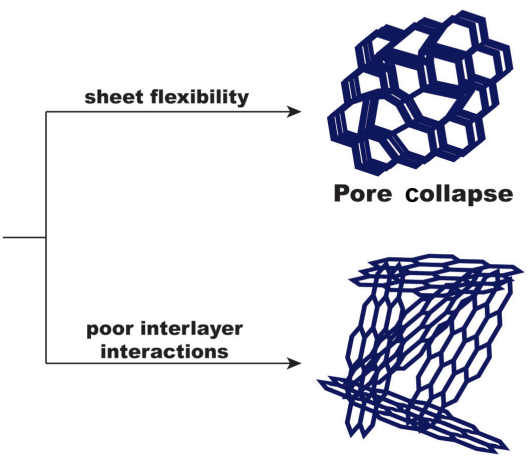

Delamination/Exfoliation

Figure 1 Inter- and/or intralayer hydrogen-bonding interactions that are available for side-chain functionalized (a) and unfunctionalized (b) hydrazone-linked COFs. (c) Illustration of pathways by which a crystalline COF can become amorphous.

activation method reported in the literature so far is solvent activation. ${ }^{24}$ Recently, more mild activation methods have been reported, which highlights the importance of proper mild activation of COFs towards their crystallinity and porosity. These methods include the activation with ultralow surface tension solvents, nitrogen-flow activation, and the use of supercritical fluid such as supercritical carbon dioxide $\left(\mathrm{scCO}_{2}\right) \cdot{ }^{24-26}$

Mechanistically, the loss of long-range order of the framework during COF formation and isolation can be associated with two possible hypotheses, random displacement of the COF layers or pore collapse during the activation process, as illustrated in Figure 1c. A recent study revealed that the displacement of COF layers upon exposure to solvent vapors causes weakening of the interlayer interactions. ${ }^{25}$ Another report has shown that hydrazone COFs can be delaminated in polar organic solvents with ultrasonication. ${ }^{18}$ Since $\mathrm{scCO}_{2}$ has a lower surface tension compared with conventional organic solvents, the strength of the capillary forces is much lower (and much less damaging) on the COF structure when it is removed. ${ }^{24-26}$

Herein, we report the synthesis and characterization of three side-chain-free hydrazone COFs that are both porous and crystalline upon $\mathrm{scCO}_{2}$ activation. Synthesis of side-

chain-free hydrazone COFs will expand the scope of hydrazone COF chemistry. To the best of our knowledge, this is the first report on $\mathrm{scCO}_{2}$ activation of hydrazone COFs, which were synthesized from unsubstituted linkers.

\section{Results and Discussion}

In this study two different tritopic linkers, 5'-(4formylphenyl)-[1,1':3',1"-terphenyl]-4,4"-dicarbaldehyde (TFPB) (Scheme S1) and 4,4',4"-(1,3,5-triazine-2,4,6-triyl) tribenzaldehyde (TFPT) (Scheme S2), and one tetratopic linker, 4,4',4",4"'-(pyrene-1,3,6,8-tetrayl)tetrabenzaldehyde (Py) (Scheme S3), were selected to synthesize hydrazone COFs with the linear ditopic linker terephthalohydrazide (DHz). The aldehyde monomers were synthesized according to previously published literature. ${ }^{18,21,27}$ Three different COFs were synthesized via the solvothermal method using optimized conditions (Scheme 1). TFPB-DHz COF and TFPTDHz COF were synthesized in a mixture of dioxane and mesitylene $(1: 9 \mathrm{v} / \mathrm{v})$ with $6 \mathrm{M}$ acetic acid as the catalyst at $120^{\circ} \mathrm{C}$ for 72 h. Py-DHz COF was synthesized in a mixture of $n$-butanol and $o$-dichlorobenzene $(1: 9 \mathrm{v} / \mathrm{v})$ with $6 \mathrm{M}$ acetic acid as the catalyst at $120^{\circ} \mathrm{C}$ for $120 \mathrm{~h}$. The activation of the COFs was carried out with both $\mathrm{scCO}_{2}$ and conventional methods. The COFs were obtained as yellow powders and were insoluble in common organic solvents such as acetone, ethanol, THF, and DMF. We synthesized a model compound (Scheme S4) for structural conformation of the synthesized COFs.

The synthesized COFs were characterized with Fourier transform infrared (FT-IR) spectroscopy. The corresponding IR spectra of COFs, monomers, and the model compound were collected (Figures S3-S5). The stretching modes observed for TFPT-DHz COF and TFPB-DHz COF at 1566-1605 and $1273 \mathrm{~cm}^{-1}$ are characteristic of $\mathrm{vC}=\mathrm{N}$ moieties, which confirms the formation of $\mathrm{C}=\mathrm{N}$ linkages in both COFs. Also, in Py-DHz COF, C $=\mathrm{N}$ stretching vibrations were observed at 1605 and $1273 \mathrm{~cm}^{-1}$. These stretching frequencies were comparable with the model compound's $\mathrm{vC}=\mathrm{N}$ stretching modes observed at 1605 and $1280 \mathrm{~cm}^{-1}$. These results indicate the formation of hydrazone moieties from the polycondensation between hydrazide and aldehyde linkers. Furthermore, the disappearance of $\mathrm{C}=\mathrm{O}$ vibrations of aldehyde monomers (at 1682, 1697, and $1689 \mathrm{~cm}^{-1}$ for TFPB, TFPT, and Py respectively) and the amino group vibration bands (at 3317 and $3209 \mathrm{~cm}^{-1}$ ) of DHz in all COFs were clear indication of the absence of starting monomers. Also, the carbonyl $(\mathrm{vC}=\mathrm{O})$ stretching vibrations of hydrazone linkage of TFPT-DHz COF, TFPBDHz COF, Py-DHz COF, model compound, and $\mathbf{D H z}$ monomer were observed at $1659,1659,1659,1651$, and $1612 \mathrm{~cm}^{-1}$ respectively. The change in the $\mathrm{VC}=0$ stretching frequency to a higher wavenumber in COFs relative to 


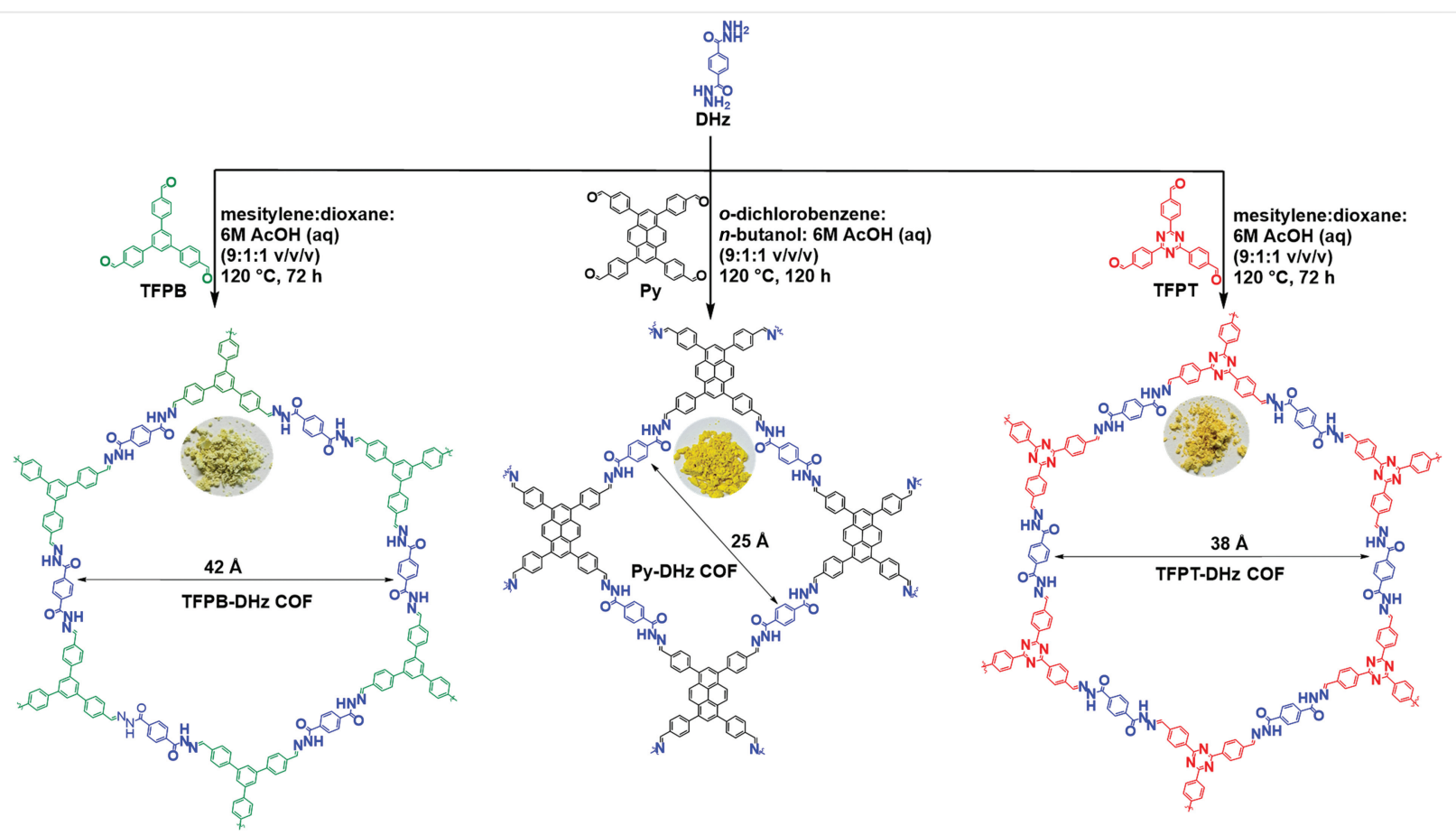

Scheme 1 Synthesis of TFPB-DHz COF, Py-DHz COF and TFPT-DHz COF through condensation of linear linker DHz with TFPB, Py and TFPT, respectively. Pore sizes shown were calculated from a computational model of the structure. Images of the COF powders are shown in the insets.

the monomer and the model compound can be attributed to the decrease in stability of the $\mathrm{C}=\mathrm{O}$ bond due to an increase in conjugation. Moreover, the $\mathrm{N}-\mathrm{H}$ stretching frequency of hydrazone moieties for TFPB-DHz COF and TFPT-DHz COF was observed at $3201 \mathrm{~cm}^{-1}$, while it was at $3178 \mathrm{~cm}^{-1}$ for Py-DHz COF. For TFPT-DHz COF, the triazine ring breath was observed at $810 \mathrm{~cm}^{-1}$, which matched with the literature reported value. ${ }^{21}$

The crystallinity of all synthesized COFs was investigated by powder X-ray diffraction (PXRD) analysis. The diffraction patterns of COFs are depicted in Figure 2. $\mathrm{scCO}_{2}$-activated TFPB-DHz COF, TFPT-DHz COF, and Py-DHz COF exhibited excellent crystallinity, while the corresponding conventional solvent activated COFs appeared to be amorphous
(Figures S8-S10). The $\mathrm{scCO}_{2}$-activated COFs showed multiple diffraction peaks in their diffraction profiles, which indicates the long-range order in the structures. In the PXRD pattern of TFPB-DHz COF, an intense diffraction peak was observed at $2.5^{\circ}$ corresponding with the (100) plane, while additional diffraction peaks were observed at $4.3^{\circ}, 5.2^{\circ}, 6.6^{\circ}$, $8.5^{\circ}, 10.0^{\circ}$, and $25.0^{\circ}$, which originated from the (200), (210), (220), (230), (310), and (001) planes, respectively (Figure 2a). Similarly, an intense peak was seen at $2.6^{\circ}$ for the TFPT-DHz COF, which corresponds to the (100) plane. In addition to the peak at $2.6^{\circ}$, multiple diffraction peaks were observed at $3.8^{\circ}, 4.3^{\circ}, 5.1^{\circ}, 6.6^{\circ}, 8.7^{\circ}, 10.5^{\circ}$, and $26.3^{\circ}$. These can be assigned to the (110), (200), (210), (220), (230), (310), and (001) facets, respectively (Figure $2 \mathrm{~b})$. The PXRD pattern
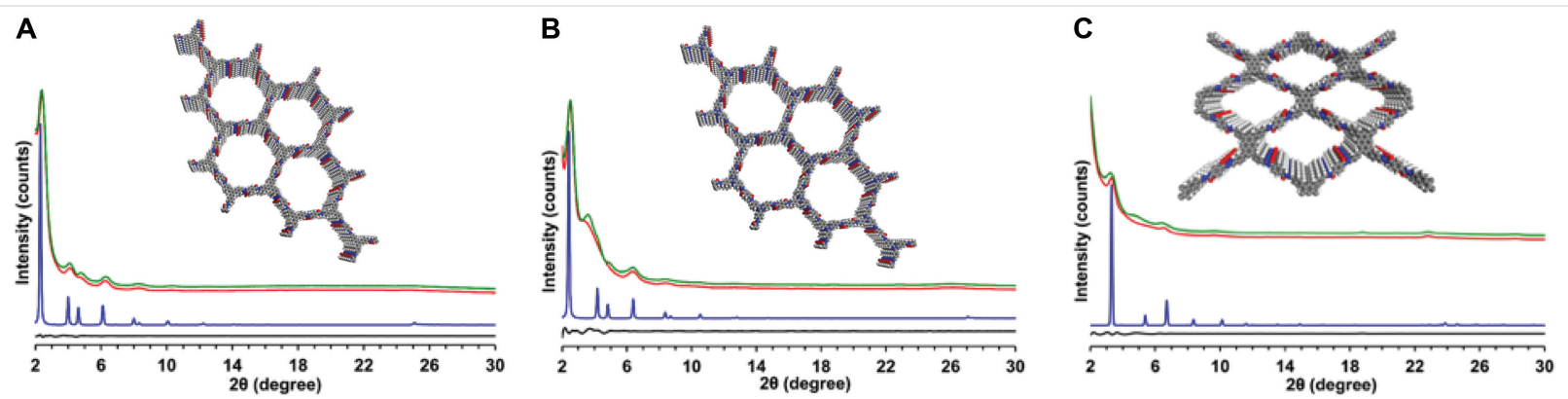

Figure 2 Experimental (green), Pawley refined (red), difference plot (black), simulated eclipsed stacking (blue) PXRD patterns of (a) TFPB-DHz COF, (b) TFPT-DHz COF, and (c) Py-DHz COF. Inset: refined AA-stacking structures. C, ash; H, white; N, blue; O, red. 
of the Py-DHz COF showed diffraction peaks at $3.3^{\circ}, 5^{\circ}$, $6.67^{\circ}, 10^{\circ}, 18.8^{\circ}, 23^{\circ}$, and $28.4^{\circ}$, which correspond to the (110), (020), (220), (330), and (001) crystal planes (Figure 2c). These results indicate that $\mathrm{scCO}_{2}$-activated COFs were highly crystalline. The interlayer distances $(d$ spacing values) corresponding to the 001 reflections were calculated to be 3.56, 3.39, and $3.15 \AA$ for TFPB-DHz COF, TFPT-DHz COF, and Py-DHz COF, respectively. This observation can be attributed to the close packing of the Py-DHz COF layers compared to the TFPB-DHz COF and TFPT-DHz COF sheets. Molecular modeling and Pawley refinements of TFPB-DHz COF, TFPT-DHz COF, and Py-DHz COF were performed using BIOVA Material Studio 2019 software package. The hexagonal lattice structural models of both TFPB-DHz COF and TFPT-DHz COF were built using the P6/ $m$ space group, with AA-eclipsed stacking model and minimized using the universal force field (UFF). The experimental PXRD patterns of both COFs had good agreement with the simulated patterns for the eclipsed stacking model. The $\left(R_{\mathrm{p}}, R_{\mathrm{wp}}\right)$ values converged to $(1.55 \%$, $2.25 \%$ ) and (3.23\%, $4.38 \%)$ for TFPB-DHz COF and TFPT-DHz COF, respectively.

The Pawley-refined unit cell parameters of the TFPBDHz COF were $a=b=45.32 \AA, c=3.65 \AA, \alpha=90^{\circ}$, $\beta=90^{\circ}$, and $\gamma=120^{\circ}$, whereas for the TFPT-DHz COF those were $\mathrm{a}=\mathrm{b}=41.14 \AA, \mathrm{c}=3.51 \AA, \alpha=90^{\circ}, \beta=90^{\circ}$, and $\gamma=120^{\circ}$. The monoclinic lattice structural model of the Py-DHz COF was built using the C2/m space group, with AAeclipsed stacking model and minimized using UFF. The experimental PXRD pattern of the Py-DHz COF had good agreement with the simulated pattern for the eclipsed stacking model. The $R_{\mathrm{p}}, R_{\mathrm{wp}}$ values converged to $2.81 \%$, $3.59 \%$, respectively. The Pawley-refined unit cell parameters of the Py-DHz COF were $\mathrm{a}=36.93 \AA, b=43.75 \AA$, c $=3.95 \AA, \alpha=\gamma=90^{\circ}$, and $\beta=119.2^{\circ}$.

The surface area of the COFs was measured by $\mathrm{N}_{2}$ adsorption experiments at $77 \mathrm{~K}$. The adsorption-desorption isotherms are shown in Figure 3. The isotherms of both TFPT-DHz COF and TFPB-DHz COF exhibited a type-IV isotherm, indicating the mesoporous nature of these materials. However, the Py-DHz COF possesses a type-I isotherm, characteristic of a microporous material. The Brunauer - Emmett - Teller (BET) surface areas of TFPTDHz COF, TFPB-DHz COF, and Py-DHz COF were found to be 1199, 790, and $932 \mathrm{~m}^{2} / \mathrm{g}$, respectively. The higher surface area of the TFPT-DHz COF compared with that of the TFPBDHz COF can be attributed to the more favorable stacking interactions in TFPT-DHz COF layers due to the highly planar structure of the triazine-centered TFPT. ${ }^{21}$ Interestingly, the conventionally activated COFs were amorphous in nature with the surface area of $55 \mathrm{~m}^{2} / \mathrm{g}$ (TFPB-DHz COF, Figure S6), $0 \mathrm{~m}^{2} / \mathrm{g}$ (TFPT-DHz COF, Figure S6), and $256 \mathrm{~m}^{2} / \mathrm{g}$ (Py-DHz COF, Figure S6). Hence this study reveals the importance of processing COFs under mild activation

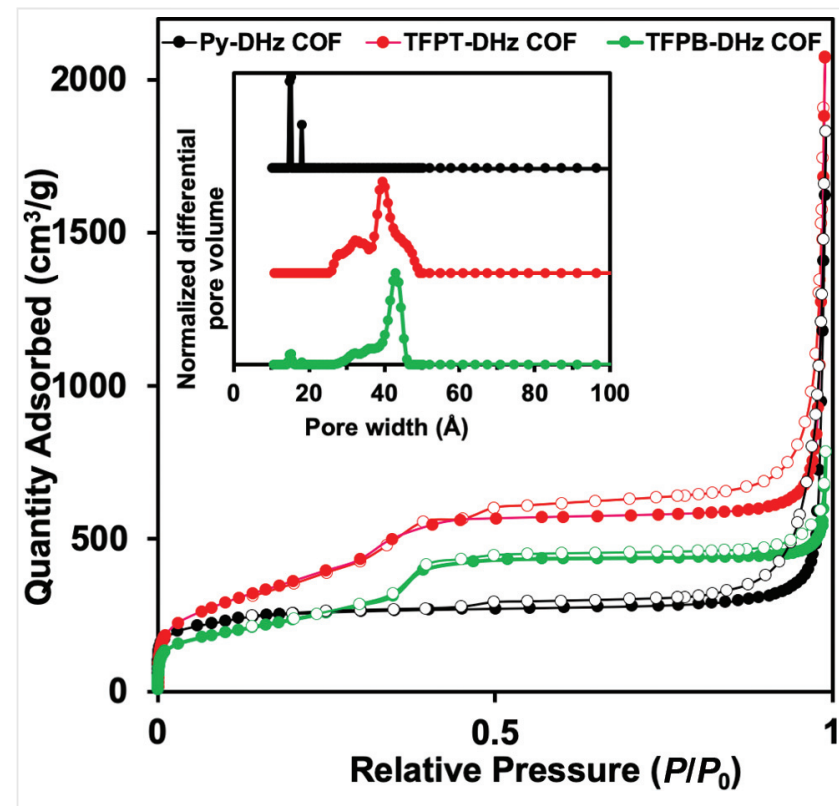

Figure $3 \mathrm{~N}_{2}$ adsorption (solid circles) and desorption (open circles) isotherms of COFs. Inset: the pore size distributions of COFs.

conditions to preserve their crystallinity and porosity. Furthermore, it indicates that the mechanism of the COF polymerization reaction is not affected by the presence of the alkoxy side chains.

The pore size distributions were determined using nonlocal density functional theory (NLDFT). The pore size distributions for each COF are shown in Figure 3. TFPB-DHz COF and TFPT-DHz COF have pore sizes confined around 37 and $41 \AA$, respectively. These pore sizes are in good agreement with the theoretical pore sizes obtained from crystal modeling of an AA-eclipsed stacking structure. The Py-DHz COF was measured to have a pore size of $17 \AA$ and showed a type I isotherm. However, the theoretical pore diameter obtained from the computational model suggests the formation of a mesoporous pore. This difference can be explained by the fact that Py-DHz COF contains non-planar tetraphenylpyrene monomer units that can experience larger offsets between the layers than a typical COF with more planarized monomer units. ${ }^{28}$

The COF polymerization process has been studied by Marder and co-workers, and they found that the disordered thin, crystalline COF layers were formed within the aggregates at the very early stage of the COF polymerization. ${ }^{16}$ Although this work was performed with imine COFs, these principles appear to be relevant in the case of hydrazone COFs, as well as hydrazone COFs, when carefully activated, retain their crystalline structure. The nature of the loss of crystallinity upon activation is less clear, especially since we have observed it with hydrazone COFs that are similar to those known to readily delaminate in 
polar solvents ${ }^{18}$ (TFPT-DHz and TFPB-DHz COFs), as well as Py-DHz COF which is made with a tetraphenylpyrene monomer that is well known to form highly correlated stacking interactions in 2D-COFs. ${ }^{29}$ It is possible that these COFs lose their crystallinity under different mechanisms (i.e., pore collapse or delamination/aggregation) depending on the type of monomer used. This will be an area of further study by our group in the future.

\section{Conclusions}

In summary, we have synthesized three different hydrazone COFs (TFPB-DHz COF, TFPT-DHz COF, and PyDHz COF) from unsubstituted hydrazide linkers. This study shows that having an alkoxy group is not essential for the formation of 2D sheets or the initial stacking of those sheets in hydrazone COFs. However, the crystallinity and porosity are significantly affected by the choice of activation method. Furthermore, by introducing the potential for greater flexibility into the COF backbone, additional functionality, such as dynamic adsorption behavior, can be imagined for hydrazone-linked COFs. We found that gentler $\mathrm{scCO}_{2}$ activation is an easy, efficient, and effective way to improve crystallinity and porosity of hydrazone COFs and may open up access to more hydrazone COF structures that were previously thought to be inaccessible.

\section{Experimental Section}

All the chemicals were purchased from commercially available sources (Fisher scientific, TCI, Acros, Alfa Aesar) and they were used in reactions as received unless otherwise mentioned. DHz was purchased from TCI. 5'(4-Formylphenyl)-[1,1':3',1"-terphenyl]-4,4"-dicarbaldehyde (TFPB) and 4,4',4"-(1,3,5-triazine-2,4,6-triyl)tribenzaldehyde (TFPT) were synthesized according to previously reported literature procedures. ${ }^{18,21}$ The ${ }^{1} \mathrm{H}$ and ${ }^{13} \mathrm{C}$ NMR spectra for all the synthesized compounds were recorded on a Bruker Avance $600 \mathrm{MHz}$ spectrometer. The FT-IR spectra of monomers, model compound, and COFs were obtained with a Cary 600 Series FT-IR spectrophotometer with an ATR attachment. The PXRD experiments were carried out using a Bruker D8 Advance diffractometer with a sealed tube radiation source ( $\mathrm{Cu} \mathrm{K} \alpha, \lambda=1.54184 \AA$ ), a low background sample holder, and a Lynxeye XE detector. Ultra-high purity grade $\mathrm{N}_{2}$ and $\mathrm{CO}_{2}$ gases were purchased from Airgas Corporation. A Micromeritics ASAP 2020 surface area analyzer was used to perform low-pressure $\mathrm{N}_{2}$ adsorptiondesorption (up to 760 torr) experiments of COFs. All analyses were carried out at $77 \mathrm{Kusing}$ a liquid $\mathrm{N}_{2}$ bath. To calculate the BET surface area of COFs, the data in the range of $0.01<P /$ $P_{0}<0.1$ were selected. The NLDFT with $\mathrm{N}_{2}$ at $77 \mathrm{~K}$ and
Cylindrical Pores in an Oxide Surface model in the Micromeritics software package was used to determine the pore size distributions of COFs. $\mathrm{scCO}_{2}$ activation was performed using a Leica EM CPD 300 Critical Point Dryer.

\section{Procedures}

\section{Synthesis of TFPB-DHz COF}

TFPB (17 mg, $0.043 \mathrm{mmol}$ ) and DHz (12.7 $\mathrm{mg}$, $0.065 \mathrm{mmol}$ ) were kept in a $5 \mathrm{~mL}$ ampoule along with mesitylene $(0.9 \mathrm{~mL})$ and dioxane $(0.1 \mathrm{~mL})$. Then, the mixture was sonicated for 10 min followed by addition of $6 \mathrm{M}$ acetic acid $(0.1 \mathrm{~mL}$, aq.). After that, the mixture was flash-frozen in liquid $\mathrm{N}_{2}$ and flame-sealed. Once the ampoule was warmed to room temperature, it was kept in an oven at $120^{\circ} \mathrm{C}$ for $72 \mathrm{~h}$ without any disturbances. Next, the ampoule was cooled to room temperature and the precipitate was collected by filtration. The resulted solid was washed with THF and ethanol. Then the wet filter cake was subjected to $\mathrm{scCO}_{2}$ activation to afford $20.4 \mathrm{mg}$ (69\%) of a colored powder of TFPB-DHz COF.

\section{Synthesis of TFPT-DHz COF}

TFPT (15 mg, $0.038 \mathrm{mmol}$ ) and DHz (11.1 mg, $0.057 \mathrm{mmol}$ ) were kept in a $5 \mathrm{~mL}$ ampoule along with mesitylene $(0.9 \mathrm{~mL})$ and dioxane $(0.1 \mathrm{~mL})$. Then the mixture was sonicated for 10 min followed by addition of $6 \mathrm{M}$ acetic acid $(0.1 \mathrm{~mL}$, aq). After that, the mixture was flash-frozen in liquid $\mathrm{N}_{2}$ and flame-sealed. Once the ampoule was warmed to room temperature, it was kept in an oven at $120{ }^{\circ} \mathrm{C}$ for $72 \mathrm{~h}$. Next, the ampoule was cooled to room temperature and the precipitate was collected by filtration. The resulted solid was washed with THF and ethanol. Then the wet filter cake was subjected to $\mathrm{scCO}_{2}$ activation to afford $14 \mathrm{mg}(54 \%)$ of a yellow colored powder of TFPT-DHz COF.

\section{Synthesis of Py-DHz COF}

Py (16 mg, $0.026 \mathrm{mmol})$ and DHz (10.1 mg, $0.052 \mathrm{mmol}$ ) were kept in a $5 \mathrm{~mL}$ ampoule along with $o$-dichlorobenzene $(0.9 \mathrm{~mL})$ and $n$-butanol $(0.1 \mathrm{~mL})$. Then the mixture was sonicated for 10 min followed by addition of $6 \mathrm{M}$ acetic acid $(0.1 \mathrm{~mL}$, aq). After that, the mixture was flash-frozen in liquid $\mathrm{N}_{2}$ and flame-sealed. Once the ampoule was warmed to room temperature, it was kept in an oven at $120^{\circ} \mathrm{C}$ for $120 \mathrm{~h}$. Next, the ampoule was cooled to room temperature and the precipitate was collected by filtration. The resulted solid was washed with THF and ethanol. Then the wet filter cake was subjected to $\mathrm{scCO}_{2}$ 
activation to afford $13.3 \mathrm{mg}$ (51\%) of a yellow colored powder of Py-DHz COF.

\section{Synthesis of Model Compound}

DHz (250 mg, $1.29 \mathrm{mmol}$ ) and benzaldehyde $(0.26 \mathrm{~mL}$, $2.57 \mathrm{mmol})$ were placed in a round bottom flask. Then, ethanol (10 mL) was added, and the mixture was refluxed for $24 \mathrm{~h}$. After that, the reaction mixture was allowed to cool to room temperature and the precipitate was filtered off. Finally, the obtained solid was washed with ethanol and vacuumdried to afford pure product as white solid (406 mg, 85\%). ${ }^{1} \mathrm{H}$ NMR(DMSO $600 \mathrm{MHz}$ ): $\delta_{\mathrm{H}} 11.99(\mathrm{~s}, 2 \mathrm{H}), 8.49(\mathrm{~s}, 2 \mathrm{H}), 8.06$ (s, $4 \mathrm{H}), 7.76(\mathrm{~d}, 4 \mathrm{H}), 7.47(\mathrm{~m}, 6 \mathrm{H}) \delta_{\mathrm{C}} 162.88,148.81,136.59$, $134.70,130.71,129.37,128.27,127.68$

\section{Supercritical Carbon Dioxide Drying of COFs}

$\mathrm{ScCO}_{2}$ activation was performed using a Leica EM CPD 300 Critical Point Dryer. After the reaction time, the COF powders were filtered and washed thoroughly with THF and ethanol. During filtration and washing, care was taken to avoid complete drying the COF powder. The wet samples were placed into the $\mathrm{sCCO}_{2}$ sample holders and were washed with $\mathrm{scCO}_{2}$ Program parameters: cooling temperature to keep $\mathrm{CO}_{2}$ fluid was $17^{\circ} \mathrm{C}$, the speed of $\mathrm{CO}_{2}$ influx in pressure chamber was set to slow, the exchange speed was set at 5 , and the number of cycles was set to 99 . The heating speed for critical point was set to medium, the temperature was set at $35^{\circ} \mathrm{C}$, and the gas release speed was set to medium.

\section{Funding Information}

This research was supported a grant from the Army Research Laboratory (W911NF-18-2-0035).

\section{Supporting Information}

Supporting Information for this article is available online at https://doi.org/10.1055/a-1477-5123.

\section{References}

(1) Côté, A. P.; Benin, A. I.; Ockwig, N. W.; O’Keeffe, M.; Matzger, A. J.; Yaghi, O. M. Science 2005, 310, 1166.

(2) Huang, N.; Wang, P.; Jiang, D. Nat. Rev. Mater. 2016, 1, 16068.

(3) Geng, K.; He, T.; Liu, R.; Dalapati, S.; Tan, K. T.; Li, Z.; Tao, S.; Gong, Y.; Jiang, Q.; Jiang, D. Chem. Rev. 2020, 120, 8814.
(4) Alahakoon, S. B.; Tan, K.; Pandey, H.; Diwakara, S. D.; McCandless, G. T.; Grinffiel, D. I.; Durand-Silva, A.; Thonhauser, T.; Smaldone, R. A. J. Am. Chem. Soc. 2020, 142, 12987.

(5) Uribe-Romo, F. J.; Doonan, C. J.; Furukawa, H.; Oisaki, K.; Yaghi, O. M. J. Am. Chem. Soc. 2011, 133, 11478.

(6) Alahakoon, S. B.; Thompson, C. M.; Nguyen, A. X.; Occhialini, G.; McCandless, G. T.; Smaldone, R. A. Chem. Commun. 2016, 52, 2843.

(7) Kuhn, P.; Antonietti, M.; Thomas, A. Angew. Chem. Int. Ed. 2008, 47, 3450.

(8) Zhang, J.; Han, X.; Wu, X.; Liu, Y.; Cui, Y.J. Am. Chem. Soc. 2017, $139,8277$.

(9) Zhao, S.; Dong, B.; Ge, R.; Wang, C.; Song, X.; Ma, W.; Wang, Y.; Hao, C.; Guo, X.; Gao, Y. RSC Adv. 2016, 6, 38774.

(10) Biswal, B. P.; Chaudhari, H. D.; Banerjee, R.; Kharul, U. K. Chem. Eur. J. 2016, 22, 4695.

(11) Alahakoon, S. B.; Thompson, C. M.; Occhialini, G.; Smaldone, R. A. ChemSusChem 2017, 10, 2116.

(12) Kaleeswaran, D.; Vishnoi, P.; Murugavel, R. J. Mater. Chem. C 2015, 3, 7159.

(13) Chen, X.; Addicoat, M.; Jin, E.; Zhai, L.; Xu, H.; Huang, N.; Guo, Z.; Liu, L.; Irle, S.; Jiang, D. J. Am. Chem. Soc. 2015, 137, 3241.

(14) Tilford, R. W.; Mugavero, S. J. III; Pellechia, P. J.; Lavigne, J. J. Adv. Mater. 2008, 20, 2741.

(15) Haase, F.; Lotsch, B. V. Chem. Soc. Rev. 2020, 49, 8469.

(16) Feriante, C.; Evans, A. M.; Jhulki, S.; Castano, I.; Strauss, M. J.; Barlow, S.; Dichtel, W. R.; Marder, S. R. J. Am. Chem. Soc. 2020, 142, 18637.

(17) Chen, G.; Lan, H.-H.; Cai, S.-L.; Sun, B.; Li, X.-L.; He, Z.-H.; Zheng, S.-R.; Fan, J.; Liu, Y.; Zhang, W.-G. ACS Appl. Mater. Interfaces 2019, 11, 12830.

(18) Bunck, D. N.; Dichtel, W. R. J. Am. Chem. Soc. 2013, 135, 14952.

(19) Qian, C.; Zhou, W.; Qiao, J.; Wang, D.; Li, X.; Teo, W. L.; Shi, X.; Wu, H.; Di, J.; Wang, H.; Liu, G.; Gu, L.; Liu, J.; Feng, L.; Liu, Y.; Quek, S. Y.; Loh, K. P.; Zhao, Y.J. Am. Chem. Soc. 2020, 142, 18138.

(20) Li, X.; Gao, Q.; Wang, J.; Chen, Y.; Chen, Z.-H.; Xu, H.-S.; Tang, W.; Leng, K.; Ning, G.-H.; Wu, J.; Xu, Q.-H.; Quek, S. Y.; Lu, Y.; Loh, K. P. Nat. Commun. 2018, 9, 2335.

(21) Stegbauer, L.; Schwinghammer, K.; Lotsch, B. V. Chem. Sci. 2014, 5,2789

(22) Li, X.; Qiao, J.; Chee, S. W.; Xu, H.-S.; Zhao, X.; Choi, H. S.; Yu, W.; Quek, S.Y.; Mirsaidov, U.; Loh, K.P.J.Am. Chem. Soc. 2020, 142, 4932.

(23) Li, J.; Peng, H.; Zhao, X.; You, N.; Wu, Y.; Wang, J.; Chen, H.; Tang, H.; Zhou, X.; Li, X.; Jiang, W. Postgrad. Med. J. 2020, 96, 742.

(24) Feriante, C. H.; Jhulki, S.; Evans, A. M.; Dasari, R. R.; Slicker, K.; Dichtel, W. R.; Marder, S. R. Adv. Mater. 2020, 32, 1905776.

(25) Sick, T.; Rotter, J. M.; Reuter, S.; Kandambeth, S.; Bach, N. N.; Döblinger, M.; Merz, J.; Clark, T.; Marder, T. B.; Bein, T.; Medina, D. D. J. Am. Chem. Soc. 2019, 141, 12570.

(26) Zhu, D.; Verduzco, R. ACS Appl. Mater. Interfaces 2020, 12, 33121.

(27) Rabbani, M. G.; Sekizkardes, A. K.; El-Kadri, O. M.; Kaafarani, B. R.; El-Kaderi, H. M. J. Mater. Chem. 2012, 22, 25409.

(28) Stegbauer, L.; Zech, S.; Savasci, G.; Banerjee, T.; Podjaski, F.; Schwinghammer, K.; Ochsenfeld, C.; Lotsch, B. V. Adv. Energy Mater. 2018, 8, 1703278.

(29) Auras, F.; Ascherl, L.; Hakimioun, A. H.; Margraf, J. T.; Hanusch, F. C.; Reuter, S.; Bessinger, D.; Döblinger, M.; Hettstedt, C.; Karaghiosoff, K.; Herbert, S.; Knochel, P.; Clark, T.; Bein, T. J. Am. Chem. Soc. 2016, 138, 16703. 